\title{
On a Theory for Analysing Second-Order Systems of Ordinary Discrete Equations
}

\author{
J. J. H. Bashingwa $\mathbb{D}^{1}$ and A. H. Kara $\mathbb{D}^{2}$ \\ ${ }^{1}$ Comp. B Group, University of Cape Town, South Africa \\ ${ }^{2}$ School of Mathematics, University of The Witwatersrand Johannesburg, South Africa \\ Correspondence should be addressed to A. H. Kara; abdul.kara@wits.ac.za
}

Received 30 December 2018; Accepted 21 April 2019; Published 12 May 2019

Academic Editor: Francisco Balibrea

Copyright (C) 2019 J. J. H. Bashingwa and A. H. Kara. This is an open access article distributed under the Creative Commons Attribution License, which permits unrestricted use, distribution, and reproduction in any medium, provided the original work is properly cited.

\begin{abstract}
We present geometric based methods for solving systems of discrete or difference equations and introduce a technique for finding conservation laws for such systems.
\end{abstract}

\section{Introduction}

Depending on a model being studied, some physical laws may be described by differential equations (DEs). Lie group theory provides us with powerful tools for obtaining analytical solutions of such equations [1]. Over the last 30 years, a considerable amount of work has been invested into applying Lie's theory to solve and classify difference equations $(\Delta \mathrm{Es})$ (see [2-6] and references therein).

The use of symmetry methods for ordinary difference equations $(\mathrm{O} \Delta \mathrm{Es})$ has been introduced by Maeda [2]. He showed that the resulting linearized symmetry condition (LSC) amounts to a set of functional equations which is hard to solve in general. Hydon introduced a technique to solve LSC and obtain symmetries in closed form by repetitive differentiations [5]. For example, a full classification of second-order $\mathrm{O} \Delta \mathrm{Es}$ according to their point symmetries exists in the literature [6]. However, for systems of difference equations (S $\Delta \mathrm{Es})$, most of results are based on induction methods (see [7-9] and references therein). In this paper, we present a method for solving $\mathrm{S} \Delta \mathrm{Es}$ using their underlying symmetry.

It has been proved in [5] that every second-order linear homogeneous $\mathrm{O} \Delta \mathrm{E}$ has an eight-dimension Lie algebra isomorphic to $\mathfrak{g l}(3)$. This is not valid for second-order system of difference equations ( $\Delta \Delta \mathrm{Es})$. We shall prove this in Section 3.

\section{Groundwork}

Let us consider an $N$-th order system of $r \Delta \mathrm{Es}$

$$
\begin{gathered}
x_{n+N}^{i}=\omega_{i}\left(n, x_{n}^{1}, \ldots, x_{n}^{r}, x_{n+1}^{1}, \ldots, x_{n+1}^{r}, \ldots, x_{n+N-1}^{1}, \ldots,\right. \\
\left.x_{n+N-1}^{r}\right), \quad i=1, \ldots, r .
\end{gathered}
$$

We assume that for each $\omega_{i}$ there exists at least one $x_{n}^{j}(i, j=$ $1, \ldots, r)$ such that $\partial \omega_{i} / \partial x_{n}^{j} \neq 0$.

Consider a point transformation

$$
\Gamma_{\epsilon}: X \longmapsto \widehat{X}(X, \epsilon)
$$

where $X=\left(x_{n}^{1}, \ldots, x_{n}^{r}\right)$ are continuous variables. $\Gamma$ will be called one-parameter Lie group of transformations if it satisfies the following properties:

(i) $\Gamma_{0}$ is the identity map, i.e., $\widehat{X}=X$ for $\epsilon=0$.

(ii) $\Gamma_{\mu} \Gamma_{\nu}=\Gamma_{\mu+\nu}$ for every $\mu$ and $\nu$ close to 0 .

(iii) Each $\widehat{x}_{n}^{i}$ can be expanded as a Taylor series in a neighbourhood of $\epsilon=0$.

Therefore, we have

$$
\begin{gathered}
\widehat{x}_{n+j}^{i}=x_{n+j}^{i}+\epsilon \mathcal{S}^{j} Q_{i}\left(n, x_{n}^{1}, \ldots, x_{n}^{r}, x_{n+1}^{1}, \ldots, x_{n+1}^{r}, \ldots,\right. \\
\left.x_{n+N-1}^{1}, \ldots, x_{n+N-1}^{r}\right)+O\left(\epsilon^{2}\right)
\end{gathered}
$$


where $Q_{i}$ are continuous functions which we shall refer to as characteristics, $i=1, \ldots, r, j=1, \ldots, N$, and $\delta$ is the "shift" operator. It is defined as follows:

$$
\begin{aligned}
\mathcal{S}: n & \longmapsto n+1, \\
\mathcal{S}^{k}\left(x_{n}^{i}\right) & =x_{n+k}^{i}
\end{aligned}
$$

We define the discrete differentiation operator as follows:

$$
\Delta=\mathcal{S}-I d
$$

where $I d$ is the identity operator.

The symmetry condition for the $\mathrm{S} \Delta \mathrm{Es}(1)$ is

$$
\begin{aligned}
& \hat{x}_{n+N}^{i}=\omega_{i}\left(n, \widehat{x}_{n}^{1}, \ldots, \widehat{x}_{n}^{r}, \widehat{x}_{n+1}^{1}, \ldots, \widehat{x}_{n+1}^{r}, \ldots, \widehat{x}_{n+N-1}^{1}, \ldots,\right. \\
& \left.\widehat{x}_{n+N-1}^{r}\right), \quad i=1, \ldots, r .
\end{aligned}
$$

whenever (1) holds.

Lie symmetries are obtained by linearizing the symmetry condition (6) about the identity. We have the following system of linearized symmetry condition (SLSC):

$$
\mathcal{S}^{N}\left(Q_{i}\right)-X \omega_{i}=0, \quad i=1, \ldots, r .
$$

where the symmetry generator $X$ is given by

$$
X=\sum_{j=0}^{N-1}\left(\sum_{i=1}^{r} \mathcal{S}^{j}\left(Q_{i}\right) \frac{\partial}{\partial x_{n+j}^{i}}\right)
$$

Definition 1. A function $w_{n}$ is invariant function under the Lie group of transformations $\Gamma$ if

$$
X\left(w_{n}\right)=0,
$$

where $w_{n}$ can be found by solving the characteristic equation

$$
\begin{aligned}
\frac{\mathrm{d} x_{n}^{1}}{Q_{1}} & =\cdots=\frac{\mathrm{d} x_{n}^{r}}{Q_{r}}=\frac{\mathrm{d} x_{n+1}^{1}}{\mathcal{S}\left(Q_{1}\right)}=\cdots=\frac{\mathrm{d} x_{n+1}^{r}}{\mathcal{S}\left(Q_{r}\right)}=\cdots . \\
& =\frac{\mathrm{d} x_{n+N-1}^{1}}{\mathcal{S}^{N-1}\left(Q_{1}\right)}=\cdots=\frac{\mathrm{d} x_{n+N-1}^{r}}{\mathcal{S}^{N-1}\left(Q_{r}\right)}=\frac{w_{n}}{0}
\end{aligned}
$$

Theorem 2. The discrete differential operator $\Delta$ in (5) and the generator of symmetry $X$ in (8) commute.

Proof. We prove the theorem for $N=1$ any generalisation is straightforward.

$$
\begin{gathered}
{[X, \Delta] F\left(n, X_{n}\right)=X\left\{\Delta\left(F\left(n, X_{n}\right)\right)\right\}} \\
-\Delta\left\{X\left(F\left(n, x_{n}^{i}\right)\right)\right\}, \\
X_{n}=\left(x_{1}, x_{2}, \ldots, x_{r}\right) \\
=X\left\{F\left(n+1, X_{n+1}\right)-F\left(n, X_{n}\right)\right\} \\
-\Delta\left(\sum_{i=1}^{r} Q_{i} \frac{\partial}{\partial x_{n}^{i}} F\left(n, X_{n}\right)\right) \\
=\sum_{i=1}^{r}\left\{\mathcal{S}\left(Q_{i}\right) \frac{\partial}{\partial x_{n+1}^{i}} F\left(n+1, X_{n+1}\right)-Q_{i} \frac{\partial}{\partial x_{n}^{i}} F\left(n, X_{n}\right)\right\} \\
-\sum_{i=1}^{r}\left\{\mathcal{S}\left(Q_{i} \frac{\partial}{\partial x_{n}^{i}} F\left(n, X_{n}\right)\right)-Q_{i} \frac{\partial}{\partial x_{n}^{i}} F\left(n, X_{n}\right)\right\} \\
=0
\end{gathered}
$$

Corollary 3. For each invariant $w_{n}$, $\delta w_{n}$ is also an invariant.

Proof. We have $X\left\{\Delta\left(w_{n}\right)\right\}=\Delta\left\{X w_{n}\right\}=0$.

Equivalently, $X\left\{(\mathcal{S}-I d) w_{n}\right\}=X\left\{\mathcal{S}\left(w_{n}\right)\right\}-X w_{n}=0$ or $X\left(\delta w_{n}\right)=0$.

We shall use this corollary for reductions in Section 3.

A first integral for the system (1) is a quantity $\phi\left(n, x_{n}, y_{n}, x_{n+1}, y_{n+1}\right)$ such that

$$
\Delta \phi\left(n, x_{n}, y_{n}, x_{n+1}, y_{n+1}\right)=0
$$

whenever (1) holds.

In Section 5, we shall use the condition (12) to develop a constructive technique for obtaining first integrals.

Remark 4. In this paper, we shall consider Lie point symmetry; i.e, the characteristics are given by $Q_{i}\left(n, x_{n}^{1}, \ldots, x_{n}^{r}\right)$.

We refer the reader to [1] for more information on symmetry methods for differential equations.

\section{Symmetries and Reductions}

3.1. Finding Characteristics. Consider a second-order system of $2 \Delta \mathrm{Es}$

$$
\begin{aligned}
& x_{n+2}=\omega_{1}\left(n, x_{n}, y_{n}, x_{n+1}, y_{n+1}\right), \\
& y_{n+2}=\omega_{2}\left(n, x_{n}, y_{n}, x_{n+1}, y_{n+1}\right) .
\end{aligned}
$$

We assume that $\partial \omega_{1} / \partial x_{n} \neq 0$ or $\partial \omega_{1} / \partial y_{n} \neq 0$ and $\partial \omega_{2} / \partial x_{n} \neq 0$ or $\partial \omega_{2} / \partial y_{n} \neq 0$, so the system is of second order. 
The SLCS (7) reduces to

$$
\begin{aligned}
& \mathcal{S}^{2}\left(Q_{1}\right)-Q_{1} \omega_{1, x_{n}}-Q_{2} \omega_{1, y_{n}}-\mathcal{S}\left(Q_{1}\right) \omega_{1, x_{n+1}} \\
& -\mathcal{S}\left(Q_{2}\right) \omega_{1, y_{n+1}}=0, \\
& \mathcal{S}^{2}\left(Q_{2}\right)-Q_{1} \omega_{2, x_{n}}-Q_{2} \omega_{2, y_{n}}-\mathcal{S}\left(Q_{1}\right) \omega_{2, x_{n+1}} \\
& -\mathcal{S}\left(Q_{2}\right) \omega_{2, y_{n+1}}=0 .
\end{aligned}
$$

where $g_{, x}=\partial g / \partial x, Q_{1}=Q_{1}\left(n, x_{n}, y_{n}\right)$, and $Q_{2}=$ $\mathrm{Q}_{2}\left(n, x_{n}, y_{n}\right)$.

The functional equations (14) and (15) contain functions $Q_{1}$ and $Q_{2}$ with different pairs of arguments making them difficult to solve. For concreteness, if, for instance, the discrete variable $n$ stands for "state" in physics, $Q_{1}\left(n, x_{n}, y_{n}\right)$ and $\mathcal{S}\left(Q_{1}\right) \equiv Q_{1}\left(n+1, x_{n+1}, y_{n+1}\right)$ belong to two different states.

To overcome this, we proceed as follows.

Step 1 (elimination of $\mathcal{S}^{2}\left(Q_{1}\right)$ and $\mathcal{S}^{2}\left(Q_{2}\right)$ ). We differentiate (total differentiation) (14) and (15) with respect to $x_{n}$ and $y_{n}$, respectively, keeping $\omega_{1}$ and $\omega_{2}$ fixed. Here, we take $x_{n+1}$ as function of $x_{n}, y_{n}, y_{n+1}, \omega_{1}, \omega_{2}$ and $y_{n+1}$ as function of $x_{n}, y_{n}, x_{n+1}, \omega_{1}, \omega_{2}$.

The total derivative operators are given by

$$
\begin{gathered}
\frac{\mathrm{d}}{\mathrm{d} x_{n}}=\frac{\partial}{\partial x_{n}}+\frac{\partial x_{n+1}}{\partial x_{n}} \frac{\partial}{\partial x_{n+1}}+\frac{\partial y_{n+1}}{\partial x_{n}} \frac{\partial}{\partial y_{n+1}}+\cdots \\
\frac{\mathrm{d}}{\mathrm{d} y_{n}}=\frac{\partial}{\partial y_{n}}+\frac{\partial x_{n+1}}{\partial y_{n}} \frac{\partial}{\partial x_{n+1}}+\frac{\partial y_{n+1}}{\partial y_{n}} \frac{\partial}{\partial y_{n+1}}+\cdots
\end{gathered}
$$

In this case, this is simplified to

$$
\begin{aligned}
\frac{\mathrm{d}}{\mathrm{d} x_{n}}= & \frac{\partial}{\partial x_{n}}-\left(\frac{\omega_{1, x_{n}}}{\omega_{1, x_{n+1}}}+\frac{\omega_{2, x_{n}}}{\omega_{2, x_{n+1}}}\right) \frac{\partial}{\partial x_{n+1}} \\
& -\left(\frac{\omega_{1, x_{n}}}{\omega_{1, y_{n+1}}}+\frac{\omega_{2, x_{n}}}{\omega_{2, y_{n+1}}}\right) \frac{\partial}{\partial y_{n+1}} \\
\frac{\mathrm{d}}{\mathrm{d} y_{n}}= & \frac{\partial}{\partial y_{n}}-\left(\frac{\omega_{1, y_{n}}}{\omega_{1, x_{n+1}}}+\frac{\omega_{2, y_{n}}}{\omega_{2, x_{n+1}}}\right) \frac{\partial}{\partial x_{n+1}} \\
& -\left(\frac{\omega_{1, y_{n}}}{\omega_{1, y_{n+1}}}+\frac{\omega_{2, y_{n}}}{\omega_{2, y_{n+1}}}\right) \frac{\partial}{\partial y_{n+1}}
\end{aligned}
$$

So we apply the operator (17) to (14) and (18) to (15) keeping $\omega_{1}$ and $\omega_{2}$ fixed. This leads to the determining system

$$
\begin{aligned}
& {\left[Q_{1} \omega_{1, x_{n}}+Q_{2} \omega_{1, y_{n}}\right]_{, x_{n}}+\mathcal{S}\left(Q_{1}\right) \omega_{1, x_{n+1}, x_{n}}+\mathcal{S}\left(Q_{2}\right)} \\
& \cdot \omega_{1, y_{n+1}, x_{n}}-\left(\frac{\omega_{1, x_{n}}}{\omega_{1, x_{n+1}}}+\frac{\omega_{2, x_{n}}}{\omega_{2, x_{n+1}}}\right)\left[Q_{1} \omega_{1, x_{n}}\right. \\
& \left.+Q_{2} \omega_{1, y_{n}}\right]_{, x_{n+1}}-\left(\frac{\omega_{1, x_{n}}}{\omega_{1, x_{n+1}}}+\frac{\omega_{2, x_{n}}}{\omega_{2, x_{n+1}}}\right) \\
& \cdot\left[\mathcal{S}\left(Q_{1}\right) \omega_{1, x_{n+1}, x_{n+1}}+\mathcal{S}\left(Q_{2}\right) \omega_{1, y_{n+1}, x_{n+1}}\right. \\
& \left.+\left[\mathcal{S}\left(Q_{1}\right)\right]_{, x_{n+1}} \omega_{1, x_{n+1}}+\left[\mathcal{S}\left(Q_{2}\right)\right]_{x_{n+1}} \omega_{1, y_{n+1}}\right]
\end{aligned}
$$

$$
\begin{aligned}
& -\left(\frac{\omega_{1, x_{n}}}{\omega_{1, y_{n+1}}}+\frac{\omega_{2, x_{n}}}{\omega_{2, y_{n+1}}}\right)\left[Q_{1} \omega_{1, x_{n}}+Q_{2} \omega_{1, y_{n}}\right]_{y_{n+1}} \\
& -\left(\frac{\omega_{1, x_{n}}}{\omega_{1, y_{n+1}}}+\frac{\omega_{2, x_{n}}}{\omega_{2, y_{n+1}}}\right)\left[\mathcal{S}\left(Q_{1}\right) \omega_{1, x_{n+1}, y_{n+1}}\right. \\
& +\mathcal{S}\left(Q_{2}\right) \omega_{1, y_{n+1}, y_{n+1}}+\left[\mathcal{S}\left(Q_{1}\right)\right]_{, y_{n+1}} \omega_{1, x_{n+1}} \\
& \left.+\left[\mathcal{S}\left(Q_{2}\right)\right]_{, y_{n+1}} \omega_{1, y_{n+1}}\right]=0,
\end{aligned}
$$

$$
\begin{aligned}
& {\left[Q_{1} \omega_{2, x_{n}}+Q_{2} \omega_{2, y_{n}}\right]_{, y_{n}}+\mathcal{S}\left(Q_{1}\right) \omega_{2, x_{n+1}, y_{n}}+\mathcal{S}\left(Q_{2}\right)} \\
& \cdot \omega_{2, y_{n+1}, y_{n}}-\left(\frac{\omega_{1, y_{n}}}{\omega_{1, x_{n+1}}}+\frac{\omega_{2, y_{n}}}{\omega_{2, x_{n+1}}}\right)\left[Q_{1} \omega_{2, x_{n}}\right. \\
& \left.+Q_{2} \omega_{2, y_{n}}\right]_{, x_{n+1}}-\left(\frac{\omega_{1, y_{n}}}{\omega_{1, x_{n+1}}}+\frac{\omega_{2, y_{n}}}{\omega_{2, x_{n+1}}}\right) \\
& +\left[\mathcal{S}\left(Q_{1}\right) \omega_{2, x_{n+1}, x_{n+1}}+\mathcal{S}\left(Q_{2}\right) \omega_{2, y_{n+1}, x_{n+1}}\right. \\
& \left.+\left[\mathcal{S}\left(Q_{1}\right)\right]_{, x_{n+1}} \omega_{2, x_{n+1}}+\left[\mathcal{S}\left(Q_{2}\right)\right]_{, x_{n+1}} \omega_{2, y_{n+1}}\right] \\
& -\left(\frac{\omega_{1, y_{n}}}{\omega_{1, y_{n+1}}}+\frac{\omega_{2, y_{n}}}{\omega_{2, y_{n+1}}}\right)\left[Q_{1} \omega_{2, x_{n}}+Q_{2} \omega_{2, y_{n}}\right]_{y_{n+1}} \\
& +\left(\frac{\omega_{1, y_{n}}}{\omega_{1, y_{n+1}}}+\frac{\omega_{2, y_{n}}}{\omega_{2, y_{n+1}}}\right)\left[\mathcal{S}\left(Q_{1}\right) \omega_{2, x_{n+1}, y_{n+1}}\right. \\
& +\mathcal{S}\left(Q_{2}\right) \omega_{2, y_{n+1}, y_{n+1}}+\left[\mathcal{S}\left(Q_{1}\right)\right]_{, y_{n+1}} \omega_{2, x_{n+1}} \\
& \left.+\left[\mathcal{S}\left(Q_{2}\right)\right]_{, y_{n+1}} \omega_{2, y_{n+1}}\right]=0 .
\end{aligned}
$$

Step 2 (elimination of $\mathcal{S}\left(Q_{1}\right)$ and $\mathcal{S}\left(Q_{2}\right)$ ). We now differentiate (19) and (20) with respect to $x_{n}$ and $y_{n}$, respectively, keeping $x_{n+1}$ and $y_{n+1}$ fixed. This means that we apply the operator $\partial / \partial x_{n}$ on (19) and $\partial / \partial y_{n}$ on (20). For a secondorder $\mathrm{S} \Delta \mathrm{Es}$ we need at most to differentiate four times. After separating with respect to $x_{n+1}$ and $y_{n+1}$ the resulting equations, we obtain a system of DEs in $Q_{1}$ and $Q_{2}$ which is solvable by hand or by using a computer algebra package.

Step 3 (explicit form of constant of integration). When integrating in Step 2 to obtain the characteristics $Q_{1}$ and $Q_{2}$, we have constant of integration which appears to be functions of $n$. To obtain their explicit form, we need to substitute the results obtained in Step 2 in (19) and (20). If we do not succeed in obtaining all the constant of integration, we need further substitution in the SLSC (14) and (15).

3.2. Reductions. Consider a second-order $\mathrm{S} \Delta \mathrm{Es}$

$$
\begin{aligned}
& x_{n+2}=\omega_{1}\left(n, x_{n}, y_{n}, x_{n+1}, y_{n+1}\right), \\
& y_{n+2}=\omega_{2}\left(n, x_{n}, y_{n}, x_{n+1}, y_{n+1}\right)
\end{aligned}
$$


and its symmetry generator

$$
X=Q_{1} \frac{\partial}{\partial x_{n}}+Q_{2} \frac{\partial}{\partial y_{n}}+\mathcal{S} Q_{1} \frac{\partial}{\partial x_{n+1}}+\mathcal{S} Q_{2} \frac{\partial}{\partial y_{n+1}}
$$

The method of characteristics for partial differential equations (PDEs)

$$
\frac{\mathrm{d} x_{n}}{\mathrm{Q}_{1}}=\frac{\mathrm{d} y_{n}}{\mathrm{Q}_{2}}=\frac{\mathrm{d} x_{n+1}}{\mathrm{Q}_{1}}=\frac{\mathrm{d} y_{n+1}}{\mathrm{Q}_{2}}=\frac{W_{n}}{0}
$$

leads to three independent constants of integration $K_{1}, K_{2}, K_{3}$. Each invariant under $X$ is function of those constant. $W_{n}=f\left(K_{1}, K_{2}, K_{3}\right)$.

For second-order systems, two invariants suffice to do reduction of the systems.

Let

$$
\begin{aligned}
& u_{n}=f_{1}\left(n, x_{n}, y_{n}, x_{n+1}, y_{n+1}\right), \\
& v_{n}=f_{2}\left(n, x_{n}, y_{n}, x_{n+1}, y_{n+1}\right)
\end{aligned}
$$

be the invariants functions under $X$. We choose them in a way that the Jacobian is nonzero.

$$
\left|\frac{\partial\left(f_{1}, f_{2}\right)}{\partial\left(x_{n+1}, y_{n+1}\right)}\right| \neq 0
$$

That is, (24) can be inverted as follows:

$$
\begin{aligned}
& x_{n+1}=g_{1}\left(n, x_{n}, y_{n}, u_{n}, v_{n}\right), \\
& y_{n+1}=g_{2}\left(n, x_{n}, y_{n}, u_{n}, v_{n}\right)
\end{aligned}
$$

By Corollary $3, \delta v_{n}$ and $\delta u_{n}$ are also invariant functions. Therefore, the solution of (21) satisfies

$$
\begin{aligned}
& u_{n+1}=\Omega_{1}\left(u_{n}, v_{n}\right), \\
& v_{n+1}=\Omega_{2}\left(u_{n}, v_{n}\right)
\end{aligned}
$$

(27) is a first-order $\mathrm{S} \triangle \mathrm{E}$ s which can be solved by further reductions or by using computer algebra software (maple, Mathematica,...) for linear systems. Note that there exist some first-order systems which cannot be solved analytically.

The general solution is

$$
\begin{aligned}
& u_{n}=u\left(n, C_{1}, C 2\right), \\
& v_{n}=v\left(n, C_{1}, C_{2}\right)
\end{aligned}
$$

for some constant $C_{1}, C_{2}$.

So the second-order system (21) is equivalent to the firstorder system obtained by substituting (28) in (26):

$$
\begin{aligned}
& x_{n+1}=g_{1}\left(n, x_{n}, y_{n}, u\left(n, C_{1}, C 2\right), v\left(n, C_{1}, C 2\right)\right), \\
& y_{n+1}=g_{2}\left(n, x_{n}, y_{n}, u\left(n, C_{1}, C 2\right), v\left(n, C_{1}, C 2\right)\right)
\end{aligned}
$$

(29) also admits the symmetries generated by $X$. The best way to integrate any first-order analytic $\Delta \mathrm{E}$ is to use its canonical coordinates [10],

$$
T_{n}=T\left(n, x_{n}, y_{n}\right)
$$

which satisfy

$$
X T_{n}=1
$$

The obvious choice of canonical coordinates is (see [10])

$$
\begin{aligned}
& t_{n}=\int \frac{\mathrm{d} x_{n}}{Q_{1}\left(n, x_{n}, y_{n}, g_{1}\left(n, x_{n}, y_{n}, u\left(n, C_{1}, C 2\right), v\left(n, C_{1}, C 2\right)\right), g_{2}\left(n, x_{n}, y_{n}, u\left(n, C_{1}, C 2\right), v\left(n, C_{1}, C 2\right)\right)\right)}, \\
& s_{n}=\int \frac{\mathrm{d} y_{n}}{Q_{2}\left(n, x_{n}, y_{n}, g_{1}\left(n, x_{n}, y_{n}, u\left(n, C_{1}, C 2\right), v\left(n, C_{1}, C 2\right)\right), g_{2}\left(n, x_{n}, y_{n}, u\left(n, C_{1}, C 2\right), v\left(n, C_{1}, C 2\right)\right)\right)}
\end{aligned}
$$

\subsection{Applications}

3.3.1. Example 1. Consider the most general homogeneous second-order linear system of difference equations

$$
\begin{aligned}
x_{n+2}= & a_{1}(n) x_{n}+a_{2}(n) y_{n}+a_{3}(n) x_{n+1} \\
& +a_{4}(n) y_{n+1}, \\
y_{n+2}= & b_{1}(n) x_{n}+b_{2}(n) y_{n}+b_{3}(n) x_{n+1}+b_{4}(n) y_{n+1}
\end{aligned}
$$

where $a_{i}(n), b_{i}(n), i=1, \ldots, 4$ are arbitrary functions.
One can readily verify that the determining system (19) and (20) amounts to

$$
\begin{aligned}
& Q_{1, x_{n} x_{n}}=Q_{2, x_{n} x_{n}}=0, \\
& Q_{1, y_{n} y_{n}}=Q_{2, y_{n} y_{n}}=0
\end{aligned}
$$

Therefore

$$
\begin{aligned}
& Q_{1}\left(n, x_{n}, y_{n}\right)=C_{1} x_{n}+C_{2} y_{n}+F_{1}(n), \\
& Q_{2}\left(n, x_{n}, y_{n}\right)=C_{3} x_{n}+C_{4} y_{n}+F_{2}(n)
\end{aligned}
$$

where $C_{i}, i=1, \ldots, 4$ are constants. 
The characteristics in (35) must satisfy the SLSC (14) and (15). Hence, we have

$$
\begin{aligned}
& F_{1}(n+2)-\left[a_{1}(n) F_{1}(n)+a_{2}(n) F_{2}(n)\right. \\
& \left.\quad+a_{3}(n) F_{1}(n+1)+a_{4}(n) F_{2}(n+1)\right]=0, \\
& F_{2}(n+2)-\left[b_{1}(n) F_{1}(n)+b_{2}(n) F_{2}(n)\right. \\
& \left.\quad+b_{3}(n) F_{1}(n+1)+b_{4}(n) F_{2}(n+1)\right]=0 .
\end{aligned}
$$

and

$$
\begin{aligned}
& C_{1}=C_{4}, \\
& C_{2}=C_{3}=0
\end{aligned}
$$

So, (35) is simplified to

$$
\begin{aligned}
& Q_{1}\left(n, x_{n}, y_{n}\right)=C_{1} x_{n}+F_{1}(n), \\
& Q_{2}\left(n, x_{n}, y_{n}\right)=C_{1} y_{n}+F_{2}(n)
\end{aligned}
$$

The first generator of symmetry for a second-order homogeneous linear system (36) is the scaling symmetry given by

$$
X=x_{n} \partial_{x_{n}}+y_{n} \partial_{y_{n}}
$$

The system (36), which governs the remaining generators of the Lie point symmetry for the system (33), is of second order in $F_{1}$ and $F_{2}$. Its general solution is

$$
\begin{aligned}
& F_{1}(n)=g_{1}\left(n, K_{1}, K_{2}, K_{3}, K_{4}\right), \\
& F_{2}(n)=g_{2}\left(n, K_{1}, K_{2}, K_{3}, K_{4}\right)
\end{aligned}
$$

where $K_{1}, i=1, \ldots, 4$ are constants.

So, the most large Lie algebra of symmetry generators which can be obtained from a homogeneous second-order system of 2 difference equations has dimension five.

For clarification let us consider

$a_{1}(n)=a_{3}(n)=a_{4}(n)=0, a_{2}(n)=1$ and $b_{2}(n)=b_{3}(n)=$ $b_{4}(n)=0, b_{1}(n)=1$. The system (33) becomes

$$
\begin{aligned}
& x_{n+2}=y_{n}, \\
& y_{n+2}=x_{n}
\end{aligned}
$$

The system which governs the remaining generators of the Lie point symmetry in this case is given by

$$
\begin{aligned}
& F_{1}(n+2)-F_{2}(n)=0, \\
& F_{2}(n+2)-F_{1}(n)=0
\end{aligned}
$$

The general solutions for this system will be

$$
\begin{array}{r}
F_{1}(n)=\frac{C_{1}\left[1+(-1)^{n}+i^{n}+(-i)^{n}\right]}{4} \\
+\frac{C_{2}\left[1-(-1)^{n}-i(i)^{n}+i(-i)^{n}\right]}{4} \\
+\frac{C_{3}\left[1+(-1)^{n}-i^{n}-(-i)^{n}\right]}{4} \\
+\frac{C_{4}\left[1-(-1)^{n}+i(i)^{n}-i(-i)^{n}\right]}{4}
\end{array}
$$

$$
\begin{array}{r}
F_{2}(n)=\frac{C_{3}\left[1+(-1)^{n}+i^{n}+(-i)^{n}\right]}{4} \\
+\frac{C_{4}\left[1-(-1)^{n}-i(i)^{n}+i(-i)^{n}\right]}{4} \\
+\frac{C_{1}\left[1+(-1)^{n}-i^{n}-(-i)^{n}\right]}{4} \\
+\frac{C_{2}\left[1-(-1)^{n}+i(i)^{n}-i(-i)^{n}\right]}{4}
\end{array}
$$

Therefore we have 5 generators of the Lie point symmetry spanned by

$$
\begin{aligned}
\mathscr{X}_{0}= & x_{n} \partial_{x_{n}}+y_{n} \partial_{y_{n}} \\
\mathscr{X}_{1}= & \frac{\left[1+(-1)^{n}+i^{n}+(-i)^{n}\right]}{4} \partial_{x_{n}} \\
& +\frac{\left[1+(-1)^{n}-i^{n}-(-i)^{n}\right]}{4} \partial_{y_{n}} \\
\mathscr{X}_{2}= & \frac{\left[1-(-1)^{n}-i(i)^{n}+i(-i)^{n}\right]}{4} \partial_{x_{n}} \\
& +\frac{\left[1-(-1)^{n}+i(i)^{n}-i(-i)^{n}\right]}{4} \partial_{y_{n}} \\
X_{3}= & \frac{\left[1+(-1)^{n}-i^{n}-(-i)^{n}\right]}{4} \partial_{x_{n}} \\
& +\frac{\left[1+(-1)^{n}+i^{n}+(-i)^{n}\right]}{4} \partial_{y_{n}} \\
\mathscr{X}_{4}= & \frac{\left[1-(-1)^{n}+i(i)^{n}-i(-i)^{n}\right]}{4} \partial_{x_{n}} \\
& +\frac{\left[1-(-1)^{n}-i(i)^{n}+i(-i)^{n}\right]}{4} \partial_{y_{n}}
\end{aligned}
$$

3.3.2. Example 2. Consider the system

$$
\begin{aligned}
& x_{n+2}=\frac{x_{n} y_{n+1}+1}{x_{n}+y_{n+1}} \\
& y_{n+2}=\frac{y_{n} x_{n+1}+1}{y_{n}+x_{n+1}}
\end{aligned}
$$

(45) is a special case of systems investigated in [11], where the author looked at the stability of the systems. 
We choose the ansatz $Q 1\left(n, x_{n}\right), Q_{2}\left(n, y_{n}\right)$.

The determining system (19) and (20) amounts to

$$
\begin{aligned}
- & Q_{2, y_{n}} x_{n+1}{ }^{2} y_{n}{ }^{2}+S Q_{1, x_{n+1}} x_{n+1}{ }^{2} y_{n}{ }^{2}+2 Q_{2} x_{n+1}{ }^{2} y_{n} \\
& -2 S Q_{1} y_{n}{ }^{2} x_{n+1}+Q_{2, y_{n}} x_{n+1}{ }^{2}+Q_{2, y_{n}} y_{n}{ }^{2} \\
& -S Q_{1, x_{n+1}} x_{n+1}{ }^{2}-S Q_{1, x_{n+1}} y_{n}{ }^{2}-2 Q_{2} y_{n} \\
& +2 S Q_{1} x_{n+1}-Q_{2, y_{n}}+S Q_{1, x_{n+1}}=0 \\
- & Q_{1, x_{n}} x_{n}{ }^{2} y_{n+1}{ }^{2}+S Q_{2, y_{n+1}} x_{n}{ }^{2} y_{n+1}{ }^{2}+2 Q_{1} y_{n+1}{ }^{2} x_{n} \\
& -2 S Q_{2} x_{n}{ }^{2} y_{n+1}+Q_{1, x_{n}} x_{n}{ }^{2}+Q_{1, x_{n}} y_{n+1}{ }^{2} \\
& -S Q_{2, y_{n+1}} x_{n}{ }^{2}-S Q_{2, y_{n+1}} y_{n+1}{ }^{2}-2 Q_{1} x_{n} \\
& +2 S Q_{2} y_{n+1}-Q_{1, x_{n}}+S Q_{2, y_{n+1}}=0
\end{aligned}
$$

Differentiating twice (46) with respect to $x_{n}$ and twice (47) with respect to $y_{n}$ keeping $x_{n+1}$ and $y_{n+1}$ fixed we obtain, after separating with respect to $x_{n+1}$ and $y_{n+1}$, the following system of Des:

$$
\begin{aligned}
& Q_{1}^{\prime \prime}+x_{n} Q_{1}^{\prime \prime \prime}+2 \frac{Q_{1}}{x_{n}{ }^{2}}-2 \frac{Q_{1}^{\prime}}{x_{n}}+\frac{Q_{1}^{\prime \prime}}{x_{n}{ }^{2}}-\frac{Q_{1}^{\prime \prime \prime}}{x_{n}}=0 \\
& Q_{2}^{\prime \prime}+y_{n} Q_{2}^{\prime \prime \prime}+2 \frac{Q_{2}}{y_{n}{ }^{2}}-2 \frac{Q_{2}^{\prime}}{y_{n}}+\frac{Q_{2}^{\prime \prime}}{y_{n}{ }^{2}}-\frac{Q_{2}^{\prime \prime \prime}}{y_{n}}=0
\end{aligned}
$$

whose most general solutions are

$$
\begin{aligned}
Q_{1}\left(n, x_{n}\right)= & F_{1}(n) x_{n}+F_{2}(n)\left(x_{n}^{2}-1\right) \ln \frac{x_{n}+1}{x_{n}-1} \\
& +F_{3}(n)\left(x_{n}^{2}-1\right) \\
Q_{2}\left(n, y_{n}\right)= & F_{4}(n) y_{n}+F_{5}(n)\left(y_{n}^{2}-1\right) \ln \frac{y_{n}+1}{y_{n}-1} \\
& +F_{6}(n)\left(y_{n}^{2}-1\right)
\end{aligned}
$$

To obtain the nature of functions $F_{1}, \ldots, F_{6}$ we substitute (49) in (46) and (47). After separating with respect to $x_{n}, x_{n+1}, y_{n}$ and $y_{n+1}$ we get the following $\mathrm{S} \triangle \mathrm{Es}$ :

$$
\begin{array}{r}
-4 F 2(n)-2 F 4(n+1)+4 F 5(n+1)-2 F 1(n)=0 \\
4 F 2(n)-2 F 4(n+1)-4 F 5(n+1)+2 F 1(n)=0 \\
-4 F 5(n)-2 F 1(n+1)+4 F 2(n+1)-2 F 4(n)=0 \\
4 F 5(n)-2 F 1(n+1)-4 F 2(n+1)+2 F 4(n)=0
\end{array}
$$

whose solutions are

$$
\begin{aligned}
& F_{1}(n)=F_{4}(n)=0 \\
& F_{2}(n)=C_{1}+(-1)^{n} C_{2} \\
& F_{5}(n)=C_{1}-(-1)^{n} C_{2}
\end{aligned}
$$

The remaining unknown functions $F_{3}(n)$ and $F_{6}(n)$ are determined by substituting (51) and (49) into the SLSC (14) and (15). This leads to the S $\triangle \mathrm{Es}$

$$
\begin{aligned}
& F_{3}(n)-F_{3}(n+2)+F_{6}(n+1)=0 \\
& F_{6}(n)+F_{3}(n+1)-F_{6}(n+2)=0
\end{aligned}
$$

The general solutions to (52) are given by

$F 3(n)$

$$
\begin{aligned}
& =\frac{1+(-1)^{n}}{\sqrt{5}}\left\{\frac{\left[(-1+\sqrt{5})^{n-1}+(1+\sqrt{5})^{n-1}\right] C_{3}}{2^{n}}\right. \\
& -\frac{\left[(-1+\sqrt{5})^{n}-(1+\sqrt{5})^{n}\right] C_{6}}{\left.2^{n+1}\right\}} \\
& \cdot \frac{-1+(-1)^{n}}{\sqrt{5}}\left\{\frac{\left[(-1+\sqrt{5})^{n-1}-(1+\sqrt{5})^{n-1}\right] C_{5}}{2^{n}}\right. \\
& \left.-\frac{\left[(-1+\sqrt{5})^{n}+(1+\sqrt{5})^{n}\right] C_{4}}{2^{n+1}}\right\}
\end{aligned}
$$

F6 (n)

$$
\begin{aligned}
& =\frac{1+(-1)^{n}}{\sqrt{5}}\left\{\frac{\left[(-1+\sqrt{5})^{n-1}+(1+\sqrt{5})^{n-1}\right] C_{5}}{2^{n}}\right. \\
& -\frac{\left[(-1+\sqrt{5})^{n}-(1+\sqrt{5})^{n}\right] C_{4}}{\left.2^{n+1}\right\}} \\
& \cdot \frac{-1+(-1)^{n}}{\sqrt{5}}\left\{\frac{\left[(-1+\sqrt{5})^{n-1}-(1+\sqrt{5})^{n-1}\right] C_{3}}{2^{n}}\right. \\
& \left.-\frac{\left[(-1+\sqrt{5})^{n}+(1+\sqrt{5})^{n}\right] C_{6}}{2^{n+1}}\right\}
\end{aligned}
$$

where $C_{1}, \ldots, C_{6}$ are arbitrary constants. It follows that the characteristics are given by

$$
\begin{aligned}
Q_{1} & =\left(C_{1}+(-1)^{n} C_{2}\right)\left(x_{n}^{2}-1\right) \ln \frac{x_{n}+1}{x_{n}-1} \\
& +\left\{\frac { 1 + ( - 1 ) ^ { n } } { \sqrt { 5 } } \left[\frac{\left[(-1+\sqrt{5})^{n-1}+(1+\sqrt{5})^{n-1}\right] C_{3}}{2^{n}}\right.\right. \\
& \left.-\frac{\left[(-1+\sqrt{5})^{n}-(1+\sqrt{5})^{n}\right] C_{6}}{2^{n+1}}\right]
\end{aligned}
$$




$$
\begin{aligned}
& \frac{-1+(-1)^{n}}{\sqrt{5}}\left[\frac{\left[(-1+\sqrt{5})^{n-1}-(1+\sqrt{5})^{n-1}\right] C_{5}}{2^{n}}\right. \\
& \left.\left.-\frac{\left[(-1+\sqrt{5})^{n}+(1+\sqrt{5})^{n}\right] C_{4}}{2^{n+1}}\right]\right\}\left(x_{n}^{2}-1\right) \\
Q_{2} & =\left(C_{1}-(-1)^{n} C_{2}\right)\left(y_{n}^{2}-1\right) \ln \frac{y_{n}+1}{y_{n}-1} \\
+ & \left\{\frac { 1 + ( - 1 ) ^ { n } } { \sqrt { 5 } } \left[\frac{\left[(-1+\sqrt{5})^{n-1}+(1+\sqrt{5})^{n-1}\right] C_{5}}{2^{n}}\right.\right. \\
& \left.-\frac{\left[(-1+\sqrt{5})^{n}-(1+\sqrt{5})^{n}\right] C_{4}}{2^{n+1}}\right] \\
& \cdot \frac{-1+(-1)^{n}}{\sqrt{5}}\left[\frac{\left[(-1+\sqrt{5})^{n-1}-(1+\sqrt{5})^{n-1}\right] C_{3}}{2^{n}}\right. \\
& \left.\left.-\frac{\left[(-1+\sqrt{5})^{n}+(1+\sqrt{5})^{n}\right] C_{6}}{2^{n+1}}\right]\right\}\left(y_{n}^{2}-1\right)
\end{aligned}
$$

Therefore, we have six generators of Lie point symmetry

$$
\begin{aligned}
X_{1} & =\left(x_{n}^{2}-1\right) \ln \frac{x_{n}+1}{x_{n}-1} \partial_{x_{n}}+\left(y_{n}^{2}-1\right) \ln \frac{y_{n}+1}{y_{n}-1} \partial_{y_{n}} \\
X_{2} & =(-1)^{n}\left(x_{n}^{2}-1\right) \ln \frac{x_{n}+1}{x_{n}-1} \partial_{x_{n}}-(-1)^{n}\left(y_{n}^{2}-1\right) \\
\cdot & \ln \frac{y_{n}+1}{y_{n}-1} \partial_{y_{n}} \\
X_{3} & =\frac{1+(-1)^{n}}{\sqrt{5}}\left[\frac{\left[(-1+\sqrt{5})^{n-1}+(1+\sqrt{5})^{n-1}\right]}{2^{n}}\right] \\
& \cdot\left(x_{n}^{2}-1\right) \partial_{x_{n}} \\
& +\frac{-1+(-1)^{n}}{\sqrt{5}}\left[\frac{\left[(-1+\sqrt{5})^{n-1}-(1+\sqrt{5})^{n-1}\right]}{2^{n}}\right] \\
& \cdot\left(y_{n}^{2}-1\right) \partial_{y_{n}} \\
x_{4} & =\frac{-1+(-1)^{n}}{\sqrt{5}}\left[\frac{\left[(-1+\sqrt{5})^{n}+(1+\sqrt{5})^{n}\right]}{2^{n+1}}\right] \\
& \cdot\left(x_{n}^{2}-1\right) \partial_{x_{n}}
\end{aligned}
$$

$$
\begin{aligned}
+ & \frac{1+(-1)^{n}}{\sqrt{5}}\left[\frac{\left[(-1+\sqrt{5})^{n}-(1+\sqrt{5})^{n}\right]}{2^{n+1}}\right] \\
\cdot\left(y_{n}^{2}-1\right) \partial_{y_{n}} & x_{5}=\frac{-1+(-1)^{n}}{\sqrt{5}}\left[\frac{\left[(-1+\sqrt{5})^{n-1}-(1+\sqrt{5})^{n-1}\right]}{2^{n}}\right] \\
\cdot\left(x_{n}^{2}-1\right) \partial_{x_{n}} & \\
+ & \frac{1+(-1)^{n}}{\sqrt{5}}\left[\frac{\left[(-1+\sqrt{5})^{n-1}+(1+\sqrt{5})^{n-1}\right]}{2^{n}}\right] \\
\cdot\left(y_{n}^{2}-1\right) \partial_{y_{n}} & \\
x_{6} & =\frac{1+(-1)^{n}}{\sqrt{5}}\left[\frac{\left[(-1+\sqrt{5})^{n}-(1+\sqrt{5})^{n}\right]}{2^{n+1}}\right] \\
& \cdot\left(x_{n}^{2}-1\right) \partial_{x_{n}} \\
& +\frac{-1+(-1)^{n}}{\sqrt{5}}\left[\frac{\left[(-1+\sqrt{5})^{n}+(1+\sqrt{5})^{n}\right]}{2^{n+1}}\right] \\
& \cdot\left(y_{n}^{2}-1\right) \partial_{y_{n}}
\end{aligned}
$$

Each generator in (55) can be used to reduce the order of (45).

Let us consider $\mathscr{X}_{1}$. By the characteristic method for Partial Differential Equations, the invariants are given by following equation:

$$
\begin{aligned}
& \frac{\mathrm{d} x_{n}}{\left(x_{n}^{2}-1\right) \ln \left(\left(x_{n}+1\right) /\left(x_{n}-1\right)\right)} \\
& \quad=\frac{\mathrm{d} y_{n}}{\left(y_{n}^{2}-1\right) \ln \left(\left(y_{n}+1\right) /\left(y_{n}-1\right)\right)} \\
& \quad=\frac{\mathrm{d} x_{n+1}}{\left(x_{n+1}^{2}-1\right) \ln \left(\left(x_{n+1}+1\right) /\left(x_{n+1}-1\right)\right)} \\
& \quad=\frac{\mathrm{d} y_{n+1}}{\left(y_{n+1}^{2}-1\right) \ln \left(\left(y_{n+1}+1\right) /\left(y_{n+1}-1\right)\right)}=\frac{V_{n}}{0}
\end{aligned}
$$

We get

$$
\begin{aligned}
& C_{1}=\frac{\ln \left(\left(x_{n}+1\right) /\left(x_{n}-1\right)\right)}{\ln \left(\left(y_{n+1}+1\right) /\left(y_{n+1}-1\right)\right)}, \\
& C_{2}=\frac{\ln \left(\left(x_{n}+1\right) /\left(x_{n}-1\right)\right)}{\ln \left(\left(y_{n}+1\right) /\left(y_{n}-1\right)\right)}, \\
& C_{3}=\frac{\ln \left(\left(x_{n}+1\right) /\left(x_{n}-1\right)\right)}{\ln \left(\left(x_{n+1}+1\right) /\left(x_{n+1}-1\right)\right)}, \\
& V_{n}=f\left(C_{1}, C_{2}, C_{3}\right)
\end{aligned}
$$

where $C_{1}, C_{2}, C_{3}$ are constants. 
If we choose $f\left(C_{1}, C_{2}, C_{3}\right)=C_{1}$, we have

$$
u_{n}=\frac{\ln \left(\left(x_{n}+1\right) /\left(x_{n}-1\right)\right)}{\ln \left(\left(y_{n+1}+1\right) /\left(y_{n+1}-1\right)\right)}
$$

and if we choose $f\left(C_{1}, C_{2}, C_{3}\right)=C_{3} / C_{2}$, we have

$$
v_{n}=\frac{\ln \left(\left(y_{n}+1\right) /\left(y_{n}-1\right)\right)}{\ln \left(\left(x_{n+1}+1\right) /\left(x_{n+1}-1\right)\right)}
$$

From (58) and (59)

we deduce

$$
\begin{aligned}
& u_{n+1}=\frac{1}{1+v_{n}} \\
& v_{n+1}=\frac{1}{1+u_{n}}
\end{aligned}
$$

Let us now consider the generator $\mathscr{X}_{3}$. The resulting invariants are

$$
\begin{aligned}
& v_{n}=\frac{\left[\left(x_{n}-1\right) /\left(x_{n}+1\right)\right]^{\alpha_{n}}}{\left(y_{n+1}-1\right) /\left(y_{n+1}+1\right)}, \\
& u_{n}=\frac{\left[\left(y_{n}-1\right) /\left(y_{n}+1\right)\right]^{\beta_{n}}}{\left(x_{n+1}-1\right) /\left(x_{n+1}+1\right)}
\end{aligned}
$$

where

$$
\begin{aligned}
& \alpha_{n}=\frac{(1+\sqrt{5})^{n}-(-1+\sqrt{5})^{n}}{2\left[(1+\sqrt{5})^{n-1}+(-1+\sqrt{5})^{n-1}\right]} \\
& \beta_{n}=\frac{(1+\sqrt{5})^{n}+(-1+\sqrt{5})^{n}}{2\left[(1+\sqrt{5})^{n-1}-(-1+\sqrt{5})^{n-1}\right]}
\end{aligned}
$$

Note also the relationship between them

$$
\begin{aligned}
& \alpha_{n+1}-1=\frac{1}{\beta_{n}}, \\
& \beta_{n+1}-1=\frac{1}{\alpha_{n}}
\end{aligned}
$$

From (61), we deduce the following relation:

$$
\begin{aligned}
& v_{n+1}=\frac{1}{u_{n}^{1 / \beta_{n}}}, \\
& u_{n+1}=\frac{1}{v_{n}^{1 / \alpha_{n}}}
\end{aligned}
$$

One can readily check that the general solution to (64) is given by

$$
\begin{aligned}
u_{n}= & \frac{1+(-1)^{n}}{2}\left[u_{0}^{\prod_{k=0}^{(n-2) / 2}\left(1 / \beta_{2 k} \alpha_{2 k+1}\right)}\right] \\
& +\frac{1-(-1)^{n}}{2}\left[v_{0}^{\prod_{k=0}^{(n-1) / 2}\left(1 / \alpha_{2 k}\right) \prod_{k=0}^{(n-3) / 2}\left(1 / \beta_{2 k+1}\right)}\right]^{-1} \\
v_{n}= & \frac{1+(-1)^{n}}{2}\left[v_{0}^{\prod_{k=0}^{(n-2) / 2}\left(1 / \alpha_{2 k} \beta_{2 k+1}\right)}\right] \\
& +\frac{1-(-1)^{n}}{2}\left[u_{0}^{\prod_{k=0}^{(n-1) / 2}\left(1 / \beta_{2 k}\right) \prod_{k=0}^{(n-3) / 2}\left(1 / \alpha_{2 k+1}\right)}\right]^{-1}
\end{aligned}
$$

where $\alpha_{n}$ and $\beta_{n}$ are defined in (62).
From (61) we obtain

$$
\begin{aligned}
& v_{n} \frac{y_{n+1}-1}{y_{n+1}+1}=\left[\frac{x_{n}-1}{x_{n}+1}\right]^{\alpha_{n}}, \\
& u_{n} \frac{x_{n+1}-1}{x_{n+1}+1}=\left[\frac{y_{n}-1}{y_{n}+1}\right]^{\beta_{n}}
\end{aligned}
$$

which is a first-order system after substitution of $u_{n}, v_{n}$ by the results given in (65). Its solutions can be obtained by using the following canonical coordinates:

$$
\begin{aligned}
& s_{n}=\ln \frac{x_{n}-1}{x_{n}+1}, \\
& t_{n}=\ln \frac{y_{n}-1}{y_{n}+1}
\end{aligned}
$$

This leads to the the following linear system with variable coefficients:

$$
\begin{aligned}
& t_{n+1}=\alpha_{n} s_{n}+\gamma_{n} \\
& s_{n+1}=\beta_{n} t_{n}+\varphi_{n}
\end{aligned}
$$

where $-\gamma_{n}=\ln u_{n}$ and $-\varphi_{n}=\ln v_{n}$.

The latter is a linear first-order system with variable coefficients. Its general solution is

$$
\begin{aligned}
s_{2 n}= & \prod_{k=0}^{n-1} \alpha_{2 k} \beta_{2 k+1} s_{0}+\sum_{r=0}^{n-1}\left(\gamma_{2 r} \prod_{j=r+1}^{n-1} \alpha_{2 j} \prod_{j=r}^{n-1} \beta_{2 j+1}\right) \\
& +\sum_{r=0}^{n-1}\left(\varphi_{2 r+1} \prod_{j=r+1}^{n-1} \alpha_{2 j} \beta_{2 j+1}\right) \\
t_{2 n}= & \prod_{k=0}^{n-1} \beta_{2 k} \alpha_{2 k+1} t_{0}+\sum_{r=0}^{n-1}\left(\varphi_{2 r} \prod_{j=r+1}^{n-1} \beta_{2 j} \prod_{j=r}^{n-1} \alpha_{2 j+1}\right) \\
& +\sum_{r=0}^{n-1}\left(\gamma_{2 r+1} \prod_{j=r+1}^{n-1} \beta_{2 j} \alpha_{2 j+1}\right) \\
t_{2 n+1}= & \prod_{k=0}^{n} \alpha_{2 k} \prod_{k=0}^{n-1} \beta_{2 k+1} s_{0}+\sum_{r=0}^{n}\left(\gamma_{2 r} \prod_{j=r+1}^{n} \alpha_{2 j} \prod_{j=r}^{n-1} \beta_{2 j+1}\right) \\
& +\sum_{r=0}^{n-1}\left(\varphi_{2 r+1} \prod_{j=r+1}^{n} \alpha_{2 j} \prod_{j=r+1}^{n-1} \beta_{2 j+1}\right) \\
& \sum_{k=0}^{n}\left(\gamma _ { 2 r + 1 } \prod _ { j = r } ^ { n - 1 } \prod _ { k = 0 } ^ { n - 1 } \alpha _ { 2 k + 1 } \beta _ { 2 j } \prod _ { j = r + 1 } ^ { n - 1 } \alpha _ { 2 j + 1 } t ^ { n } \left(\sum_{r=0}^{n}\left(\varphi_{2 r} \prod_{j=r+1}^{n} \beta_{2 j} \prod_{j=r}^{n-1} \alpha_{2 j+1}\right)\right.\right. \\
s_{2 n+1} & \\
& =
\end{aligned}
$$

The general solution of (45) is obtained by substituting (69) into (67). 


\section{Conservation Laws}

In Section 2, we have defined a first integral associated with a second-oreder $\mathrm{S} \triangle \mathrm{E}$. It is given by (12)

$$
\phi\left(n, x_{n}, y_{n}, x_{n+1}, y_{n+1}\right)=\phi\left(n+1, x_{n+1}, y_{n+1}, \omega_{1}, \omega_{2}\right)
$$

Let

$$
\begin{aligned}
P_{1} & =\frac{\partial \phi}{\partial x_{n}}, \\
P_{2} & =\frac{\partial \phi}{\partial x_{n+1}} \\
Q_{1} & =\frac{\partial \phi}{\partial y_{n}}, \\
Q_{2} & =\frac{\partial \phi}{\partial y_{n+1}}
\end{aligned}
$$

By differentiating (70) with respect to $x_{n}, y_{n}, x_{n+1}$ and $y_{n+1}$ we obtain

$$
\begin{aligned}
& P_{1}=\mathcal{S}\left(P_{2}\right) \omega_{1, x_{n}}+\mathcal{S}\left(Q_{2}\right) \omega_{2, x_{n}} \\
& Q_{1}=\mathcal{S}\left(P_{2}\right) \omega_{1, y_{n}}+\mathcal{S}\left(Q_{2}\right) \omega_{2, y_{n}}
\end{aligned}
$$

and

$$
\begin{aligned}
P_{2} & =\mathcal{S}\left(P_{1}\right)+\mathcal{S}\left(P_{2}\right) \omega_{1, x_{n+1}}+\mathcal{S}\left(Q_{2}\right) \omega_{2, x_{n+1}} \\
Q_{2} & =\mathcal{S}\left(Q_{1}\right)+\mathcal{S}\left(P_{2}\right) \omega_{1, y_{n+1}}+\mathcal{S}\left(Q_{2}\right) \omega_{2, y_{n+1}}
\end{aligned}
$$

The substitution of (72) in (73) leads to the following secondorder system of functional equations:

$$
\begin{aligned}
& \mathcal{S}^{2}\left(P_{2}\right) \mathcal{S}\left(\omega_{1, x_{n}}\right)+\mathcal{S}^{2}\left(Q_{2}\right) \mathcal{S}\left(\omega_{2, x_{n}}\right) \\
& +\mathcal{S}\left(P_{2}\right) \omega_{1, x_{n+1}}+\mathcal{S}\left(Q_{2}\right) \omega_{2, x_{n+1}}-P_{2}=0 \\
& \mathcal{S}^{2}\left(P_{2}\right) \mathcal{S}\left(\omega_{1, y_{n}}\right)+\mathcal{S}^{2}\left(Q_{2}\right) \mathcal{S}\left(\omega_{2, y_{n}}\right) \\
& \quad+\mathcal{S}\left(P_{2}\right) \omega_{1, y_{n+1}}+\mathcal{S}\left(Q_{2}\right) \omega_{2, y_{n+1}}-Q_{2}=0
\end{aligned}
$$

As for SLSC, we differentiate repeatedly to obtain a system of DEs for $P_{2}$ and $Q_{2}$. Given the solutions $P_{2}, Q_{2}$ of (74) we easily construct $P_{1}, Q_{1}$. For consistency of our solutions, we must check the integrability conditions

$$
\frac{\partial P_{1}}{\partial x_{n+1}}=\frac{\partial P_{2}}{\partial x_{n}}
$$

and

$$
\frac{\partial Q_{1}}{\partial y_{n+1}}=\frac{\partial Q_{2}}{\partial y_{n}}
$$

The first integral is then given by

$$
\phi=\int\left(P_{1} \mathrm{~d} x_{n}+P_{2} \mathrm{~d} x_{n+1}+Q_{1} \mathrm{~d} y_{n}+Q_{2} \mathrm{~d} y_{n+1}\right)+F(n)
$$

The constant of integration $F(n)$ which is a function depending on $n$ is determined by substituting (77) in (70).

4.1. Applications. Let us consider the second-order $\mathrm{S} \Delta \mathrm{Es}$

$$
\begin{aligned}
& x_{n+2}=a(n) y_{n}, \\
& y_{n+2}=b(n) x_{n}
\end{aligned}
$$

By choosing the ansatz $P_{2}\left(n, x_{n}, y_{n}\right)$ and $Q_{2}\left(n, x_{n}, y_{n}\right)$ one can readily check that the determining system (74) is simplified to

$$
\begin{aligned}
& Q_{2}\left(n+2, \omega_{1}, \omega_{2}\right) b(n+1)-P_{2}\left(n, x_{n}, y_{n}\right)=0, \\
& P_{2}\left(n+2, \omega_{1}, \omega_{2}\right) a(n+1)-Q_{2}\left(n, x_{n}, y_{n}\right)=0 .
\end{aligned}
$$

where $\omega_{1}$ and $\omega_{2}$ denote the right-hand side of (78).

Differentiating (79) with respect to $x_{n}$ and $y_{n}$ leads to

$$
\begin{gathered}
P_{2}=x_{n} \psi_{1}(n)+y_{n} \psi_{2}(n)+\psi_{3}(n), \\
Q_{2}=x_{n} \psi_{4}(n)+y_{n} \psi_{5}(n)+\psi_{6}(n)
\end{gathered}
$$

Thus, we have from (72)

$$
\begin{aligned}
& P_{1} \\
& =b(n)\left[x_{n+1} \psi_{4}(n+1)+y_{n+1} \psi_{5}(n+1)+\psi_{6}(n+1)\right] \\
& Q_{1} \\
& =a(n)\left[x_{n+1} \psi_{1}(n+1)+y_{n+1} \psi_{2}(n+1)+\psi_{3}(n+1)\right]
\end{aligned}
$$

Substituting (80) in (79) and separating with respect to $x_{n}$ and $y_{n}$ we obtain the system

$$
\begin{array}{r}
a(n) b(n+1) \psi_{4}(n+2)-\psi_{2}(n)=0, \\
b(n) a(n+1) \psi_{2}(n+2)-\psi_{4}(n)=0, \\
b(n) b(n+1) \psi_{5}(n+2)-\psi_{1}(n)=0, \\
a(n) a(n+1) \psi_{1}(n+2)-\psi_{5}(n)=0, \\
b(n+1) \psi_{6}(n+2)-\psi_{3}(n)=0, \\
a(n+1) \psi_{3}(n+2)-\psi_{6}(n)=0 .
\end{array}
$$

The solutions to (82) will provide us with the explicit form of $\psi_{i}, i=1, \ldots, 6$

The first integral is then given by 
$\phi$

$$
\begin{aligned}
& =\int b(n)\left[x_{n+1} \psi_{4}(n\right. \\
& \left.+1)+y_{n+1} \psi_{5}(n+1)+\psi_{6}(n+1)\right] \mathrm{d} x_{n} \\
& +\left(x_{n} \psi_{1}(n)+y_{n} \psi_{2}(n)+\psi_{3}(n)\right) \mathrm{d} x_{n+1} a(n)\left[x_{n+1} \psi_{1}(n+1)+y_{n+1} \psi_{2}(n+1)+\psi_{3}(n+1)\right] \mathrm{d} y_{n} \\
& +\left(x_{n} \psi_{4}(n)+y_{n} \psi_{5}(n)+\psi_{6}(n)\right) \mathrm{d} y_{n+1} \\
& +K_{i}
\end{aligned}
$$

for some constants $K_{i}$.

For clarification, let us consider $a(n)=b(n)=1$, that is,

$$
\begin{aligned}
& x_{n+2}=y_{n}, \\
& y_{n+2}=x_{n}
\end{aligned}
$$

The solutions to (82) will be

$$
\begin{aligned}
\psi_{1}(n)= & \frac{C_{1}\left[1+(-1)^{n}+i^{n}+(-i)^{n}\right]}{4} \\
& +\frac{C_{2}\left[1-(-1)^{n}-i(i)^{n}+i(-i)^{n}\right]}{4} \\
& +\frac{C_{3}\left[1+(-1)^{n}-i^{n}-(-i)^{n}\right]}{4} \\
\psi_{5}(n)= & \frac{C_{3}\left[1+(-1)^{n}+i^{n}+(-i)^{n}\right]}{4} \\
& +\frac{C_{4}\left[1-(-1)^{n}+i(i)^{n}-i(-i)^{n}\right]}{4} \\
& +\frac{C_{1}\left[1-(-1)^{n}-i(i)^{n}+i(-i)^{n}\right]}{4} \\
& +\frac{C_{8}\left[1-(-1)^{n}-i^{n}-(-i)^{n}\right]}{4} \\
\psi_{2}(n)= & \frac{C_{5}\left[1+(-1)^{n}+i^{n}+(-i)^{n}\right]}{4} \\
& +i\left(1+(-1)^{n}-i(-i)^{n}\right] \\
4 & C_{6}\left[1-(-1)^{n}-i(i)^{n}+i(-i)^{n}\right] \\
4 &
\end{aligned}
$$

$$
\begin{aligned}
\psi_{4}(n)= & \frac{C_{7}\left[1+(-1)^{n}+i^{n}+(-i)^{n}\right]}{4} \\
& +\frac{C_{8}\left[1-(-1)^{n}-i(i)^{n}+i(-i)^{n}\right]}{4} \\
& +\frac{C_{5}\left[1+(-1)^{n}-i^{n}-(-i)^{n}\right]}{4} \\
& +\frac{C_{6}\left[1-(-1)^{n}+i(i)^{n}-i(-i)^{n}\right]}{4}
\end{aligned}
$$$$
\psi_{3}(n)=\frac{C_{9}\left[1+(-1)^{n}+i^{n}+(-i)^{n}\right]}{4}
$$$$
+\frac{C_{10}\left[1-(-1)^{n}-i(i)^{n}+i(-i)^{n}\right]}{4}
$$$$
+\frac{C_{11}\left[1+(-1)^{n}-i^{n}-(-i)^{n}\right]}{4}
$$$$
+\frac{C_{12}\left[1-(-1)^{n}+i(i)^{n}-i(-i)^{n}\right]}{4}
$$$$
\psi_{6}(n)=\frac{C_{11}\left[1+(-1)^{n}+i^{n}+(-i)^{n}\right]}{4}
$$$$
+\frac{C_{12}\left[1-(-1)^{n}-i(i)^{n}+i(-i)^{n}\right]}{4}
$$$$
+\frac{C_{9}\left[1+(-1)^{n}-i^{n}-(-i)^{n}\right]}{4}
$$$$
+\frac{C_{10}\left[1-(-1)^{n}+i(i)^{n}-i(-i)^{n}\right]}{4}
$$

where $C_{i}, i=1, \ldots, 12$ are constants. We have twelve solutions for $P_{2}$ and $Q_{2}$. That is,

(1) If

$$
\begin{aligned}
P_{2} & =\left(\frac{\left[1+(-1)^{n}+i^{n}+(-i)^{n}\right]}{4}\right) x_{n}, \\
Q_{2} & =\left(\frac{\left[1+(-1)^{n}-i^{n}-(-i)^{n}\right]}{4}\right) y_{n},
\end{aligned}
$$


then

$$
\begin{aligned}
& Q_{1}=\left(\frac{\left[1-(-1)^{n}+i i^{n}-i(-i)^{n}\right]}{4}\right) x_{n+1}, \\
& P_{1}=\left(\frac{\left[1-(-1)^{n}-i i^{n}+i(-i)^{n}\right]}{4}\right) y_{n+1}
\end{aligned}
$$

(2) If

$$
\begin{aligned}
& P_{2}=\left(\frac{\left[1-(-1)^{n}-i(i)^{n}+i(-i)^{n}\right]}{4}\right) x_{n}, \\
& Q_{2}=\left(\frac{\left[1-(-1)^{n}+i(i)^{n}-i(-i)^{n}\right]}{4}\right) y_{n},
\end{aligned}
$$

then

$$
\begin{aligned}
& Q_{1}=\left(\frac{\left[1+(-1)^{n}+(i)^{n}+(-i)^{n}\right]}{4}\right) x_{n+1}, \\
& P_{1}=\left(\frac{\left[1+(-1)^{n}-(i)^{n}-(-i)^{n}\right]}{4}\right) y_{n+1}
\end{aligned}
$$

(3) If

$$
\begin{gathered}
\left.P_{2}=\frac{\left[1+(-1)^{n}-i^{n}-(-i)^{n}\right]}{4}\right) x_{n}, \\
Q_{2}=\left(\frac{\left[1+(-1)^{n}+i^{n}+(-i)^{n}\right]}{4}\right) y_{n},
\end{gathered}
$$

then

$$
\begin{aligned}
& Q_{1}=\left(\frac{\left[1-(-1)^{n}-i i^{n}+i(-i)^{n}\right]}{4}\right) x_{n+1}, \\
& P_{1}=\left(\frac{\left[1-(-1)^{n}+i i^{n}-i(-i)^{n}\right]}{4}\right) y_{n+1}
\end{aligned}
$$

(4) If

$$
\begin{aligned}
& P_{2}=\left(\frac{\left[1-(-1)^{n}+i(i)^{n}-i(-i)^{n}\right]}{4}\right) x_{n}, \\
& Q_{2}=\left(\frac{\left[1-(-1)^{n}-i(i)^{n}+i(-i)^{n}\right]}{4}\right) y_{n},
\end{aligned}
$$

then

$$
\begin{aligned}
& Q_{1}=\left(\frac{\left[1+(-1)^{n}-(i)^{n}-(-i)^{n}\right]}{4}\right) x_{n+1}, \\
& P_{1}=\left(\frac{\left[1+(-1)^{n}+(i)^{n}+(-i)^{n}\right]}{4}\right) y_{n+1}
\end{aligned}
$$

(5) If

$$
\begin{aligned}
P_{2} & =\left(\frac{\left[1+(-1)^{n}+i^{n}+(-i)^{n}\right]}{4}\right) y_{n}, \\
Q_{2} & =\left(\frac{\left[1+(-1)^{n}-i^{n}-(-i)^{n}\right]}{4}\right) x_{n},
\end{aligned}
$$

then

$$
\begin{gathered}
Q_{1}=\left(\frac{\left[1-(-1)^{n}+i i^{n}-i(-i)^{n}\right]}{4}\right) y_{n+1}, \\
P_{1}=\left(\frac{\left[1-(-1)^{n}-i i^{n}+i(-i)^{n}\right]}{4}\right) x_{n+1}
\end{gathered}
$$

(6) If

$$
\begin{array}{r}
P_{2}=\left(\frac{\left[1-(-1)^{n}-i(i)^{n}+i(-i)^{n}\right]}{4}\right) y_{n}, \\
Q_{2}=\left(\frac{\left[1-(-1)^{n}+i(i)^{n}-i(-i)^{n}\right]}{4}\right) x_{n},
\end{array}
$$

then

$$
\begin{gathered}
Q_{1}=\left(\frac{\left[1+(-1)^{n}+(i)^{n}+(-i)^{n}\right]}{4}\right) y_{n+1}, \\
P_{1}=\left(\frac{\left[1+(-1)^{n}-(i)^{n}-(-i)^{n}\right]}{4}\right) x_{n+1}
\end{gathered}
$$

(7) If

$$
\begin{gathered}
\left.P_{2}=\frac{\left[1+(-1)^{n}-i^{n}-(-i)^{n}\right]}{4}\right) y_{n}, \\
Q_{2}=\left(\frac{\left[1+(-1)^{n}+i^{n}+(-i)^{n}\right]}{4}\right) x_{n},
\end{gathered}
$$

then

$$
\begin{gathered}
Q_{1}=\left(\frac{\left[1-(-1)^{n}-i i^{n}+i(-i)^{n}\right]}{4}\right) y_{n+1}, \\
P_{1}=\left(\frac{\left[1-(-1)^{n}+i i^{n}-i(-i)^{n}\right]}{4}\right) x_{n+1}
\end{gathered}
$$

(8) If

$$
\begin{array}{r}
P_{2}=\left(\frac{\left[1-(-1)^{n}+i(i)^{n}-i(-i)^{n}\right]}{4}\right) y_{n}, \\
Q_{2}=\left(\frac{\left[1-(-1)^{n}-i(i)^{n}+i(-i)^{n}\right]}{4}\right) x_{n},
\end{array}
$$


then

$$
\begin{aligned}
& Q_{1}=\left(\frac{\left[1+(-1)^{n}-(i)^{n}-(-i)^{n}\right]}{4}\right) y_{n+1}, \\
& P_{1}=\left(\frac{\left[1+(-1)^{n}+(i)^{n}+(-i)^{n}\right]}{4}\right) x_{n+1}
\end{aligned}
$$

(9) If

$$
\begin{array}{r}
P_{2}=\left(\frac{\left[1+(-1)^{n}+i^{n}+(-i)^{n}\right]}{4}\right), \\
Q_{2}=\left(\frac{\left[1+(-1)^{n}-i^{n}-(-i)^{n}\right]}{4}\right),
\end{array}
$$

then

$$
\begin{gathered}
Q_{1}=\left(\frac{\left[1-(-1)^{n}+i i^{n}-i(-i)^{n}\right]}{4}\right), \\
P_{1}=\left(\frac{\left[1-(-1)^{n}-i i^{n}+i(-i)^{n}\right]}{4}\right)
\end{gathered}
$$

(10) If

$$
\begin{aligned}
P_{2} & =\left(\frac{\left[1-(-1)^{n}-i(i)^{n}+i(-i)^{n}\right]}{4}\right), \\
Q_{2} & =\left(\frac{\left[1-(-1)^{n}+i(i)^{n}-i(-i)^{n}\right]}{4}\right),
\end{aligned}
$$

then

$$
\begin{gathered}
Q_{1}=\left(\frac{\left[1+(-1)^{n}+(i)^{n}+(-i)^{n}\right]}{4}\right), \\
P_{1}=\left(\frac{\left[1+(-1)^{n}-(i)^{n}-(-i)^{n}\right]}{4}\right)
\end{gathered}
$$

(11) If

$$
\begin{gathered}
\left.P_{2}=\frac{\left[1+(-1)^{n}-i^{n}-(-i)^{n}\right]}{4}\right), \\
Q_{2}=\left(\frac{\left[1+(-1)^{n}+i^{n}+(-i)^{n}\right]}{4}\right),
\end{gathered}
$$

then

$$
\begin{gathered}
Q_{1}=\left(\frac{\left[1-(-1)^{n}-i i^{n}+i(-i)^{n}\right]}{4}\right), \\
P_{1}=\left(\frac{\left[1-(-1)^{n}+i i^{n}-i(-i)^{n}\right]}{4}\right)
\end{gathered}
$$

(12) If

$$
\begin{array}{r}
P_{2}=\left(\frac{\left[1-(-1)^{n}+i(i)^{n}-i(-i)^{n}\right]}{4}\right), \\
Q_{2}=\left(\frac{\left[1-(-1)^{n}-i(i)^{n}+i(-i)^{n}\right]}{4}\right),
\end{array}
$$

then

$$
\begin{aligned}
Q_{1} & =\left(\frac{\left[1+(-1)^{n}-(i)^{n}-(-i)^{n}\right]}{4}\right), \\
P_{1} & =\left(\frac{\left[1+(-1)^{n}+(i)^{n}+(-i)^{n}\right]}{4}\right)
\end{aligned}
$$

Therefore, we obtain twelve conservation laws for the system (84). They are given by

$$
\begin{aligned}
& \phi_{1}=\left(\frac{\left[1+(-1)^{n}+i^{n}+(-i)^{n}\right]}{4}\right) x_{n} x_{n+1} \\
& +\left(\frac{\left[1+(-1)^{n}-i^{n}-(-i)^{n}\right]}{4}\right) y_{n} y_{n+1} \\
& +\left(\frac{\left[1-(-1)^{n}+i i^{n}-i(-i)^{n}\right]}{4}\right) x_{n+1} y_{n} \\
& +\left(\frac{\left[1-(-1)^{n}-i i^{n}+i(-i)^{n}\right]}{4}\right) y_{n+1} x_{n} \\
& +K_{1} \\
& \phi_{2}=\left(\frac{\left[1-(-1)^{n}-i(i)^{n}+i(-i)^{n}\right]}{4}\right) x_{n} x_{n+1} \\
& +\left(\frac{\left[1-(-1)^{n}+i(i)^{n}-i(-i)^{n}\right]}{4}\right) y_{n} y_{n+1} \\
& +\left(\frac{\left[1+(-1)^{n}+(i)^{n}+(-i)^{n}\right]}{4}\right) x_{n+1} y_{n} \\
& +\left(\frac{\left[1+(-1)^{n}-(i)^{n}-(-i)^{n}\right]}{4}\right) y_{n+1} x_{n} \\
& +K_{2} \\
& \left.\phi_{3}=\frac{\left[1+(-1)^{n}-i^{n}-(-i)^{n}\right]}{4}\right) x_{n} x_{n+1} \\
& +\left(\frac{\left[1+(-1)^{n}+i^{n}+(-i)^{n}\right]}{4}\right) y_{n} y_{n+1} \\
& +\left(\frac{\left[1-(-1)^{n}-i i^{n}+i(-i)^{n}\right]}{4}\right) x_{n+1} y_{n} \\
& +\left(\frac{\left[1-(-1)^{n}+i i^{n}-i(-i)^{n}\right]}{4}\right) y_{n+1} x_{n} \\
& +K_{3}
\end{aligned}
$$

$$
\begin{aligned}
\phi_{4}= & \left(\frac{\left[1-(-1)^{n}+i(i)^{n}-i(-i)^{n}\right]}{4}\right) x_{n} x_{n+1} \\
& +\left(\frac{\left[1-(-1)^{n}-i(i)^{n}+i(-i)^{n}\right]}{4}\right) y_{n} y_{n+1} \\
& +\left(\frac{\left[1+(-1)^{n}-(i)^{n}-(-i)^{n}\right]}{4}\right) x_{n+1} y_{n} \\
& +\left(\frac{\left[1+(-1)^{n}+(i)^{n}+(-i)^{n}\right]}{4}\right) y_{n+1} x_{n}
\end{aligned}
$$$$
+K_{4}
$$ 


$$
\begin{aligned}
& \phi_{5}=\left(\frac{\left[1+(-1)^{n}+i^{n}+(-i)^{n}\right]}{4}\right) y_{n} x_{n+1} \\
& +\left(\frac{\left[1+(-1)^{n}-i^{n}-(-i)^{n}\right]}{4}\right) x_{n} y_{n+1} \\
& +\left(\frac{\left[1-(-1)^{n}+i i^{n}-i(-i)^{n}\right]}{4}\right) y_{n+1} y_{n} \\
& +\left(\frac{\left[1-(-1)^{n}-i i^{n}+i(-i)^{n}\right]}{4}\right) x_{n+1} x_{n} \\
& +K_{5} \\
& \phi_{6}=\left(\frac{\left[1-(-1)^{n}-i(i)^{n}+i(-i)^{n}\right]}{4}\right) y_{n} x_{n+1} \\
& +\left(\frac{\left[1-(-1)^{n}+i(i)^{n}-i(-i)^{n}\right]}{4}\right) x_{n} y_{n+1} \\
& +\left(\frac{\left[1+(-1)^{n}+(i)^{n}+(-i)^{n}\right]}{4}\right) y_{n+1} y_{n} \\
& +\left(\frac{\left[1+(-1)^{n}-(i)^{n}-(-i)^{n}\right]}{4}\right) x_{n+1} x_{n} \\
& +K_{6} \\
& \left.\phi_{7}=\frac{\left[1+(-1)^{n}-i^{n}-(-i)^{n}\right]}{4}\right) y_{n} x_{n+1} \\
& +\left(\frac{\left[1+(-1)^{n}+i^{n}+(-i)^{n}\right]}{4}\right) x_{n} y_{n+1} \\
& +\left(\frac{\left[1-(-1)^{n}-i i^{n}+i(-i)^{n}\right]}{4}\right) y_{n+1} y_{n} \\
& +\left(\frac{\left[1-(-1)^{n}+i i^{n}-i(-i)^{n}\right]}{4}\right) x_{n+1} x_{n} \\
& +K_{7} \\
& \phi_{8}=\left(\frac{\left[1-(-1)^{n}+i(i)^{n}-i(-i)^{n}\right]}{4}\right) y_{n} x_{n+1} \\
& \phi_{9}=\left(\frac{\left[1+(-1)^{n}+i^{n}+(-i)^{n}\right]}{4}\right) x_{n+1} \\
& +\left(\frac{\left[1+(-1)^{n}-i^{n}-(-i)^{n}\right]}{4}\right) y_{n+1} \\
& +\left(\frac{\left[1-(-1)^{n}+i i^{n}-i(-i)^{n}\right]}{4}\right) y_{n} \\
& +\left(\frac{\left[1-(-1)^{n}-i i^{n}+i(-i)^{n}\right]}{4}\right) x_{n}+K_{9} \\
& \phi_{10}=\left(\frac{\left[1-(-1)^{n}-i(i)^{n}+i(-i)^{n}\right]}{4}\right) x_{n+1} \\
& +\left(\frac{\left[1-(-1)^{n}+i(i)^{n}-i(-i)^{n}\right]}{4}\right) y_{n+1} \\
& +\left(\frac{\left[1+(-1)^{n}+(i)^{n}+(-i)^{n}\right]}{4}\right) y_{n} \\
& +\left(\frac{\left[1+(-1)^{n}-(i)^{n}-(-i)^{n}\right]}{4}\right) x_{n}+K_{10} \\
& \left.\phi_{11}=\frac{\left[1+(-1)^{n}-i^{n}-(-i)^{n}\right]}{4}\right) x_{n+1} \\
& +\left(\frac{\left[1+(-1)^{n}+i^{n}+(-i)^{n}\right]}{4}\right) y_{n+1} \\
& +\left(\frac{\left[1-(-1)^{n}-i i^{n}+i(-i)^{n}\right]}{4}\right) y_{n} \\
& +\left(\frac{\left[1-(-1)^{n}+i i^{n}-i(-i)^{n}\right]}{4}\right) x_{n}+K_{11} \\
& \phi_{12}=\left(\frac{\left[1-(-1)^{n}+i(i)^{n}-i(-i)^{n}\right]}{4}\right) x_{n+1} \\
& +\left(\frac{\left[1-(-1)^{n}-i(i)^{n}+i(-i)^{n}\right]}{4}\right) y_{n+1} \\
& +\left(\frac{\left[1+(-1)^{n}-(i)^{n}-(-i)^{n}\right]}{4}\right) y_{n} \\
& +\left(\frac{\left[1+(-1)^{n}+(i)^{n}+(-i)^{n}\right]}{4}\right) x_{n}+K_{12}
\end{aligned}
$$$$
+\left(\frac{\left[1-(-1)^{n}-i(i)^{n}+i(-i)^{n}\right]}{4}\right) x_{n} y_{n+1}
$$$$
+\left(\frac{\left[1+(-1)^{n}-(i)^{n}-(-i)^{n}\right]}{4}\right) y_{n+1} y_{n}
$$$$
+\left(\frac{\left[1+(-1)^{n}+(i)^{n}+(-i)^{n}\right]}{4}\right) x_{n+1} x_{n}
$$$$
+K_{8}
$$

\section{Conclusion and Discussions}

We have presented a method for obtaining nontrivial symmetries and how to use them for solving a second-order $\mathrm{S} \Delta \mathrm{Es}$. Each symmetry can be used to reduce the order. However, different symmetries lead to different reductions (see (60) and (64)), but the same solution. We also proposed a technique to construct first integral associated to second-order systems of difference equations. 


\section{Data Availability}

No data were used to support this study.

\section{Conflicts of Interest}

The authors declare that they have no conflicts of interest.

\section{References}

[1] P. J. Olver, Applications of Lie Groups to Differential Equations, Springer, New York, NY, USA, 1993.

[2] S. Maeda, "The similarity method for difference equations," IMA Journal of Applied Mathematics, vol. 38, no. 2, pp. 129-134, 1987.

[3] D. Levi, L. Vinet, and P. Winternitz, "Lie group formalism for difference equations," Journal of Physics A: Mathematical and General, vol. 30, no. 2, pp. 633-649, 1997.

[4] G. R. W. Quispel and R. Sahadevan, "Lie symmetries and the integration of difference equations," Physics Letters A, vol. 184, no. 1, pp. 64-70, 1993.

[5] P. E. Hydon, Difference Equations by Differential Equation Methods, Cambridge University Press, Cambridge, 2014.

[6] V. Dorodnitsyn, R. Kozlov, and P. Winternitz, "Lie group classification of second-order ordinary difference equations," Journal of Mathematical Physics, vol. 41, no. 1, pp. 480-504, 2000.

[7] N. Touafek and E. M. Elsayed, "On the solutions of systems of rational difference equations," Mathematical and Computer Modelling, vol. 55, no. 7-8, pp. 1987-1997, 2012.

[8] E. M. Elsayed and T. F. Ibrahim, "Periodicity and solutions for some systems of nonlinear rational difference equations," Hacettepe Journal of Mathematics and Statistics, vol. 44, no. 6, pp. 1361-1390, 2015.

[9] A. S. Kurbanl, C. Çinar, and I. Yalçinkaya, "On the behavior of positive solutions of the system of rational difference equations $x_{n+1}=x_{n-1} /\left(y_{n} x_{n-1}+1\right), y_{n+1}=y_{n-1} /\left(x_{n} y_{n-1}+1\right), "$ Mathematical and Computer Modelling, vol. 53, no. 5-6, pp. 1261-1267, 2011.

[10] N. Joshi and P. J. Vassiliou, "The existence of Lie symmetries for first-order analytic discrete dynamical systems," Journal of Mathematical Analysis and Applications, vol. 195, no. 3, pp. 872887, 1995.

[11] I. Yalcinkaya, "On the global asymptotic stability of a second order system of difference equation," Discrete Dynamics in Nature and Society, vol. 2008, Article ID 860152, 12 pages, 2008. 


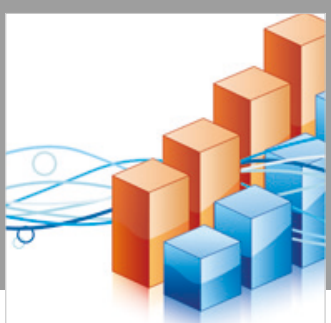

Advances in

Operations Research

\section{-n-m}
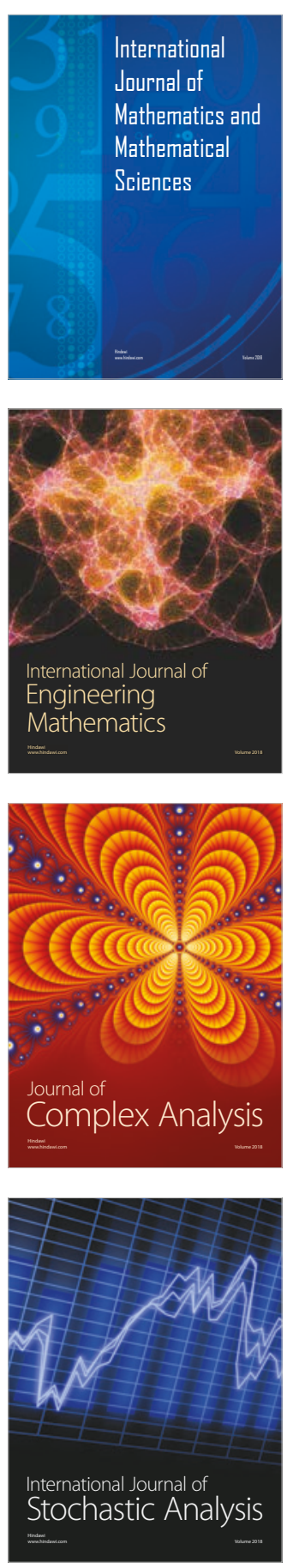
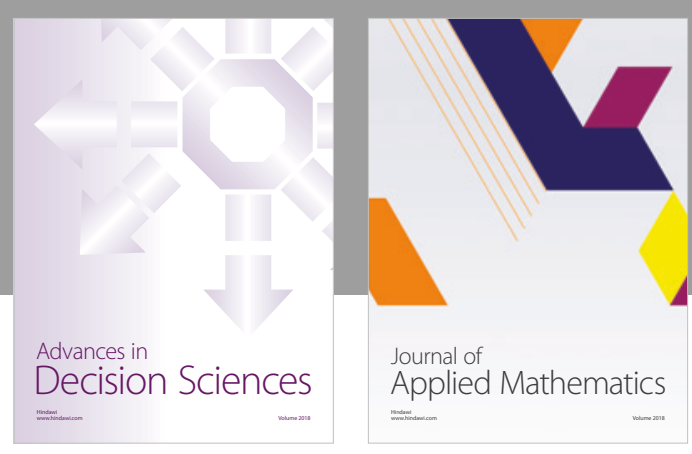

Journal of

Applied Mathematics
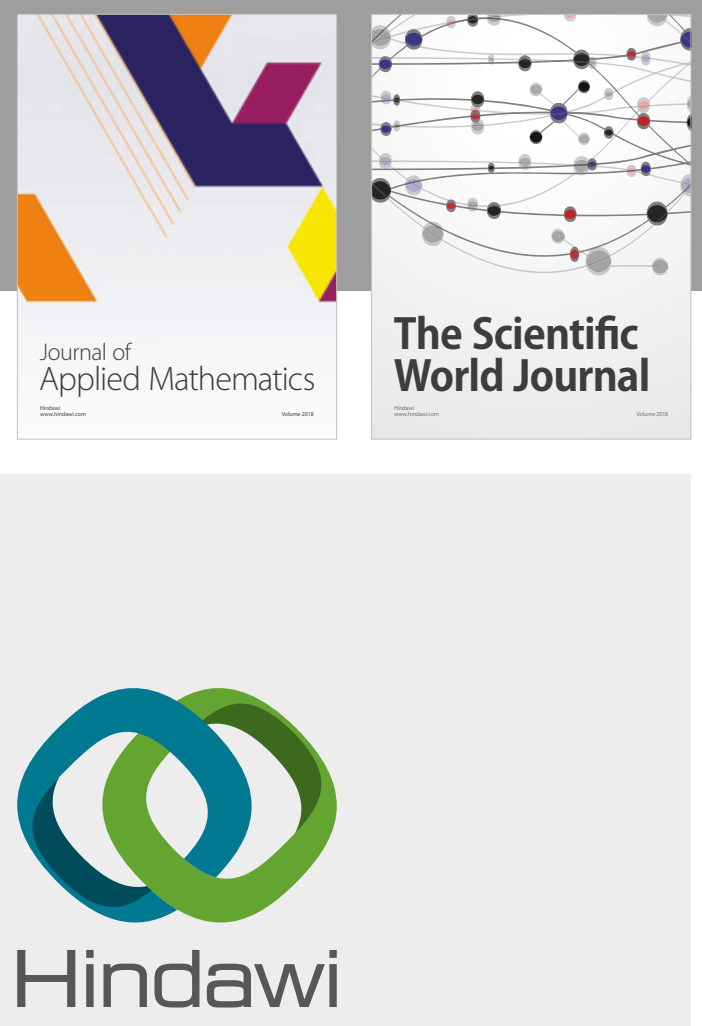

Submit your manuscripts at

www.hindawi.com

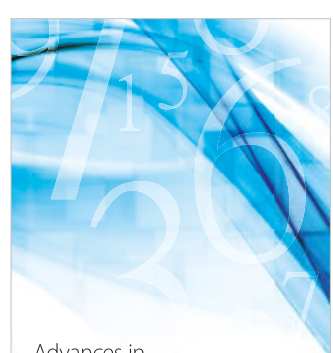

Advances in
Numerical Analysis
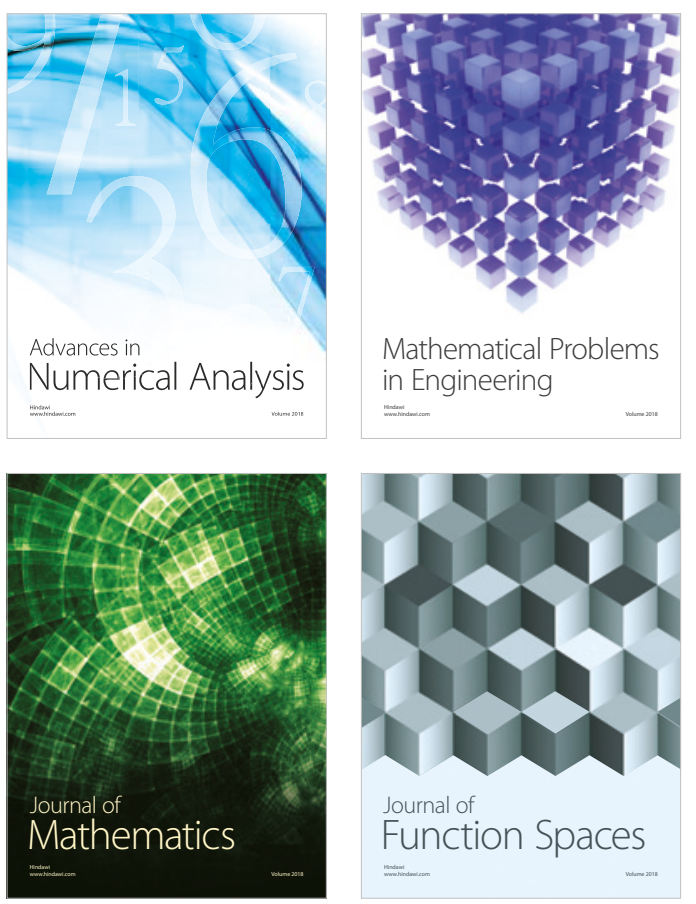

Mathematical Problems in Engineering

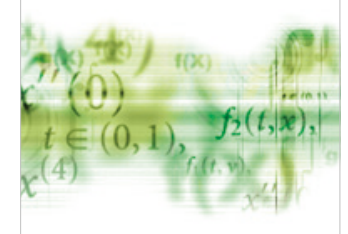

International Journal of

Differential Equations

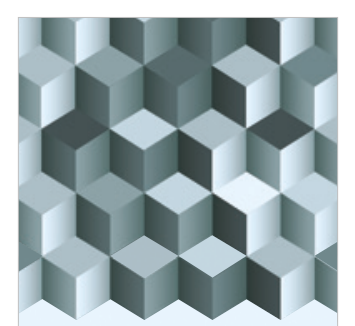

Journal of

Function Spaces

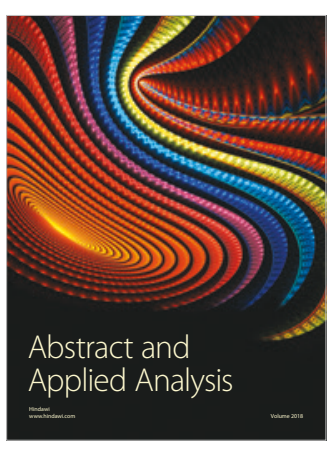

The Scientific

World Journal

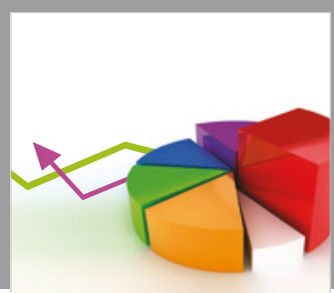

Journal of

Probability and Statistics
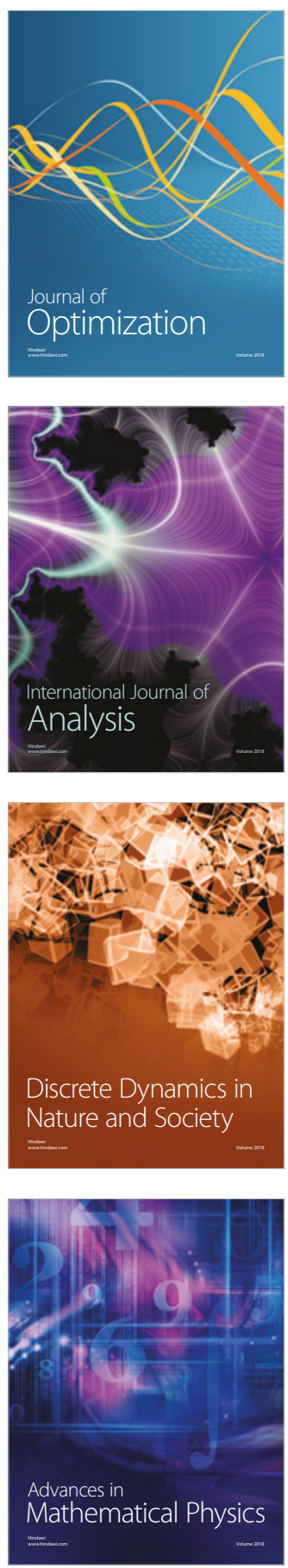